Memorias del VII Encuentro Nacional de Experiencias en la Enseñanza de la Biología y la Educación Ambiental y II Congreso Nacional de Investigación en la Enseñanza de la Biología

\title{
A PROPÓSITO DE LAS REPRESENTACIONES SOCIALES DE AMBIENTE, Y SU INCIDENCIA EN EL PLAN INSTITUCIONAL DE GESTIÓN AMBIENTAL DEL JARDÍN BOTÁNICO JOSÉ CELESTINO MUTIS, BOGOTÁ
}

\section{Edna Marisol Gil Torres ${ }^{1}$ Yarmiht Yurany González Roa ${ }^{2}$}

\section{Durante miles de años el hombre \\ luchó por abrirse un lugar en la naturaleza. \\ Por primera vez en la historia de nuestra especie, la situación se ha invertido y hoy es indispensable hacerle un lugar a la naturaleza en el mundo del hombre. Santiago Kavadlof}

\section{Resumen}

En esta investigación se identifican las representaciones sociales de ambiente de los funcionarios del Jardín Botánico de Bogotá en el plan institucional de gestión ambiental de esta entidad. Se desarrolla utilizando tres instrumentos: encuesta, carta de asociación de palabras y soportes gráficos, en donde los funcionarios manifiestan su postura frente al ambiente. El análisis se efectuó a través de categorías previas, tomadas de las tipologías de ambiente propuestas por Reigota (1999) quién destaca cinco: antropocéntricas culturales, pactuadas y utilitaristas; naturalistas y globalizantes. Además las autoras colocan a disposición de la comunidad científica dos nuevas categorías: la emotiva y la cromática, que surgen del análisis de los instrumentos. En conclusión, se identifico que predominan las representaciones sociales de ambiente de corte naturalista y que la forma de actuar de quienes diseñan, elaboran y ejecutan el PIGA, parte de su conocimiento,

\footnotetext{
${ }^{1}$ Licenciada en Biología, de la Universidad Pedagógica Nacional, Colombia.Magister en Ciencias de la Educación de la Universidad San Buenaventura, Colombia. Docente de Ecología y Recursos Naturales de la Universidad de Cundinamarca, Docente de Investigación en Especializaciones de la Universidad Juan de Castellanos, Tunja Boyacá, Colombia.

${ }^{2}$ Licenciada en Biología, de la Universidad Pedagógica Nacional, Colombia. Especialista en Gerencia de Instituciones Educativas de la Universidad del Tolima, Especialista en Pedagogía de la Recreación Ecológica de la Universidad Los Libertadores, Colombia, Especialista en Gestión Ambiental de la Fundación Universitaria del Área Andina, Colombia, Magister en Ciencias de la Educación de la Universidad San Buenaventura, Colombia.. Docente de Biología de la Secretaria de Educación del Distrito, Docente de Investigación en Especializaciones de la Universidad Juan de Castellanos, Tunja Boyacá, Colombia.
} 
Bio-grafía Escritos sobre la Biología y su Enseñanza.

Edición Extra-Ordinaria. ISSN 2027-1034 P.p 95 - 103

Memorias del VII Encuentro Nacional de Experiencias en la Enseñanza de la Biologia y la Educación Ambiental y II Congreso Nacional de Investigación en la Enseñanza de la Biología

labor y contexto frente al ambiente, es decir a las representaciones que tengan del mismo.

Palabras claves: Representaciones Sociales, Ambiente, Piga, educación ambiental, desarrollo sostenible.

\section{Abstract}

In this research we identify the social representations of concept environment with officials Bogota Botanical Garden, under the institutional plan environmental management of this entity. It develops through three instruments where officials expressed their position on the environment: survey letter word association and supports graphics. The analysis was performed through previous categories, taken from the environment typologies proposed by Reigota (1999) who highlights five: anthropocentric cultural and utilitarian pactuadas, naturalists and globalizing. In addition, the authors placed at the disposal of the scientific community two new categories: the emotional and the color from the analysis of the instruments. In conclusion, it was found that dominant social representations naturalistic environment and the modus operandi of those who design develop and implement the PIGA, part of his knowledge, work and context from the environment, ie the representations are the same.

Key words: Social Representations, Environment, Piga, environmental education, sustainable development.

\section{Introducción}

La siguiente, es una síntesis del trabajo de investigación desarrollado desde el grupo TAEPE adscrito a Colciencias, llamado Tendencias Actuales en Educación Y Pedagogía, cuya entidad promotora es la Universidad San Buenaventura. Se estructura en tres secciones fundamentales; en la parte inicial se esbozan las generalidades de las Representaciones Sociales, (en adelante RS) y del Plan Institucional de Gestión Ambiental (en adelante PIGA), además se puntualiza el problema de investigación, en la segunda parte se mencionan los objetivos y la metodología aplicada, y finalmente se hace una descripción de los resultados y la discusión de estos.

Colombia en su condición de segundo país megabiodiverso del planeta debe asumir la responsabilidad de formar comunidades planetarias comprometidas con criterios de conservación, uso racional y sostenibilidad de los recursos, así, la sociedad se centra en la búsqueda de actitudes que permiten pensar, sentir y 
Memorias del VII Encuentro Nacional de Experiencias en la Enseñanza de la Biología y la Educación Ambiental y II Congreso Nacional de Investigación en la Enseñanza de la Biología

actuar ambientalmente, en este sentido en el año 2006 el Departamento Administrativo del Medio Ambiente DAMA hoy la Secretaria Distrital de Ambiente SDA, crea estrategias que contribuyen a la formación de la cultura ambiental para todas las entidades del Distrito Capital, con la formulación del -PIGA - el cual, pretende promover el análisis y reconocimiento de los problemas y potencialidades ambientales, creando espacios de participación en los que interviene la comunidad, para desarrollar y proponer soluciones acordes con las dinámicas naturales y socioculturales.

Ahora bien, Se precisa para la presente investigación el concepto de RS desde la definición que ofrece Serge Moscovisci (1979. págs. 75-79) como una modalidad particular del conocimiento, cuya función es la elaboración de los comportamientos y la comunicación entre los individuos. Las Representaciones Sociales surgen en determinada comunidad y se manifiestan frente a situaciones concretas, en este caso el ambiente.

\section{Metodología}

Así, el problema central de esta investigación surge al pensar que por ser el Jardín botánico la entidad considerada como el aula ambiental abierta máxima de Bogotá, y centro científico de asesoramiento con fines educativos de investigación y experimentación, a la cual acuden la mayoría de instituciones educativas de todos los niveles, y toman como referente en criterios de educación ambiental, se hace necesario entonces, determinar desde qué concepciones de ambiente se gestan y desarrollan los planes y proyectos que desde allí se direccionan a todas las localidades del distrito capital y que impactan en la construcción de cultura ambiental en la comunidad.

De esta manera el objetivo central de esta investigación es Caracterizar las representaciones sociales de ambiente que tienen los funcionarios del Jardín Botánico de Bogotá, puesto que son ellos los que viabilizan, interpretan, ejecutan y desarrollan el Plan Institucional de Gestión Ambiental; y como objetivos específicos se propone 1) Identificar y describir las representaciones sociales de ambiente presentes en sus funcionarios, 2) Interpretar el PIGA de esta institución y finalmente 3) relacionarlo con las RSA encontradas. EI PIGA es un instrumento que debe señalar las acciones de gestión ambiental a desarrollar por las entidades distritales para prevenir y mitigar los daños al ambiente, haciendo uso ecoeficiente y sostenible de los recursos. Es importante señalar y justificar 
Bio-grafía Escritos sobre la Biología y su Enseñanza.

Edición Extra-Ordinaria. ISSN 2027-1034 P. p 95 - 103

Memorias del VII Encuentro Nacional de Experiencias en la Enseñanza de la Biologia y la Educación Ambiental y II Congreso Nacional de Investigación en la Enseñanza de la Biología

que el PIGA en sí mismo es un instrumento que fomenta la educación ambiental en las instituciones educativas y no educativas, por lo tanto su talante pedagógico es intrínseco y en efecto válido para éste estudio.

La presente es una investigación cualitativa- Interpretativa de enfoque interdisciplinar, la técnica aplicable es el análisis de contenido, que se hace a partir de la aplicación de tres instrumentos a los funcionarios del JBB, a saber a)encuesta, b) carta de asociación de palabras y c) soportes gráficos, que indagan por su concepto de ambiente. A partir de la información obtenida, se realiza una caracterización apoyados en un referente teórico. Esta institución cuenta con 700 funcionarios que se desempeñan en cuatro divisiones; administrativa, científica, educativa- cultural y técnica - operativa. Para determinar la muestra se tomó el 20 \% de la población de forma aleatoria.

En este orden de ideas y cumpliendo con los objetivos, se interpretó el PIGA del JBB, permitiendo finalmente señalar la relación de las Representaciones Sociales de Ambiente con el PIGA del JBB.

Los resultados se muestran tomando como base la categorización propuesta por Marcos Reigota en 1999 quien señalo tres categorías de representaciones sociales de ambiente: naturalista, globalizante y antropocéntrica.

\section{Resultados y Discusión}

A continuación se presentan los principales hallazgos:

En cuanto a la Categoría Naturalista: la mayoría de funcionarios pertenecientes a esta categoría relacionan la palabra ambiente con ecosistema y naturaleza, dibujan paisajes donde predominan plantas, animales y ríos sin incluir figuras humanas.

En la Categoría Antropocéntrica los funcionarios relacionan la palabra ambiente con sociedad, educación, cultura, recursos, sustento y contaminación, evidencian además imágenes donde el hombre hace uso del ambiente y a su vez lo deteriora.

En la Categoría Globalizante los funcionarios relacionan la palabra ambiente con planeta, interrelación, sostenibilidad, equilibrio y dibujan imágenes que sugieren la relación del hombre con la cultura, la sociedad y su entorno. 
Bio-grafía Escritos sobre la Biología y su Enseñanza.

Edición Extra-Ordinaria. ISSN 2027-1034 P. p 95 - 103

Memorias del VII Encuentro Nacional de Experiencias en la Enseñanza de la Biologia y la Educación Ambiental y II Congreso Nacional de Investigación en la Enseñanza de la Biología

Así mismo con base en la interpretación de los instrumentos las investigadoras se permiten presentar además dos nuevas categorías a la comunidad educativa Estas son: CATEGORÍA EMOTIVA Y CATEGORÍA CROMÁTICA, estas generan nuevos aportes a la teoría relacionada con los estudios en representaciones sociales de ambiente, las cuales, se describen tanto en el campo representacional como en el campo de la información, basándose en fundamentos teóricos tan importantes que van desde la teoría evolutiva de Darwin en el caso de la categoría emotiva hasta la teoría del color de Eva Heller (España, 2008) en la categoría cromática, los hallazgos que las sustentan son:

Categoría Emotiva: los funcionarios relacionan la palabra ambiente con armonía, bienestar, vitalidad, alegría y paz, donde se permite espacios de reencuentro, reconciliación e introspección, lo que le da la posibilidad de sentirse sujeto ecológico en perfecta fraternidad con el entorno.

En la Categoría Cromática los funcionarios relacionan la palabra ambiente con colores como: el verde, amarillo, rojo, blanco, azul entre otros. Se percibe una visión del ambiente desde la estética y el encuentro del sujeto con su entorno a partir de los sentidos y las emociones que pueden generar en él, la visualización de los colores y cómo estos se traducen en diferentes sensaciones.

\section{Reflexiones Finales}

En la apuesta investigativa se propuso caracterizar las representaciones sociales de ambiente y su importancia en el PIGA, este proceso dio como resultado, el reconocimiento de una visión naturalista en los funcionarios que interactúan en el JBB. Es decir, el ambiente es entendido como naturaleza, como sitio donde se desarrollan un determinado tipo de seres vivos; y un conjunto de factores abióticos que influyen en los organismos. Además, se privilegian los componentes de fauna y de flora por encima de la especie humana; lo anterior, admite una estrecha relación con la formación académica de las personas que laboran en el JBB, sus deseos, sus ideas, la manera como ven su contexto, la comprensión que tienen de ambiente, y la forma de entender la realidad del planeta.

Cabe anotar, que la percepción de ambiente planteada por el JBB desde el PIGA, corresponde a una perspectiva que incluye factores naturales, sociales y culturales; lo que permite, comprender los procesos globales. 


\begin{abstract}
Bio-grafia Escritos sobre la Biología y su Enseñanza.
Edición Extra-Ordinaria. ISSN 2027-1034 P. p 95 - 103

Memorias del VII Encuentro Nacional de Experiencias en la Enseñanza de la Biologia y la Educación Ambiental y II Congreso Nacional de Investigación en la Enseñanza de la Biología
\end{abstract}

En conclusión, se ratifica una mayor incidencia de la categoría Naturalista y, en consecuencia, la discusión sobre la concepción de ambiente y especialmente sobre educación ambiental, es ineludible, ya que al hacerlo se permiten adaptar nociones más reflexivas y menos tradicionales desde el punto de vista ético, histórico, social, cultural y político. De tal forma, que las RSA deben ser la materia prima para la implementación y puesta en marcha del PIGA, en todas las instituciones del D.C, y así, lograr un cambio real en el pensar y actuar del hombre en beneficio del planeta.

Es importante resaltar que no se evidencio una estricta coherencia entre lo escrito en el PIGA y los resultados que se encontraron al caracterizar las RSA de los funcionarios de esta entidad, así que, el JBB como máxima autoridad a nivel ambiental en la ciudad, debe aplicar estrategias que conlleven a cambios en la concepción de ambiente en sus funcionarios, que se manifiesten en los proyectos que implementan, teniendo como base la educación ambiental y la bioética para que conduzcan a la formación de sujetos con conciencia ecológica. Se debe recordar que somos parte de un planeta moldeado al antojo humano, desbordado en tecnología y conocimiento, pero carente de conciencia ambiental. Se requiere de manera urgente formar ciudadanos reconciliados con el planeta, que construyan espacios de sana y armónica convivencia con el entorno, cumpliendo con los pilares de los PIGA.

Con la mirada que hacen las investigadoras desde su labor docente se reconoce la necesidad de una escuela que se vincule activamente con estas entidades para que haga su aporte significativo en la formación de humanos más humanos, conscientes, responsables y respetuosos consigo mismo, con los demás seres vivos y con su entorno, la escuela en todos los niveles debe formar personas con actitud crítica, reflexiva, responsable y autónoma capaces de responder a las exigencias del entorno cambiante, globalizado y competitivo, por eso debe formar procesos respetuosos hacia la vida donde la relación entre persona, ciencia y tecnología sean sinónimo de convivencia holística, desarrollo, cultura y progreso. De este modo la educación ambiental puede convertirse en la esperanza que tiene este planeta para sobrevivir a la crisis y la escuela es la encargada hacerlo realidad.

\title{
Referencias
}


Bio-grafia Escritos sobre La Biología y su Enseñanza.

Edición Extra-Ordinaria. ISSN 2027-1034 P. p 95 - 103

Memorias del VII Encuentro Nacional de Experiencias en la Enseñanza de la Biologia y la Educación Ambiental y II Congreso Nacional de Investigación en la Enseñanza de la Biología

Alarcón, F. (2004). Las representaciones sociales de las unidades de servicio de apoyo a la educación popular. Bellaterra.

Andreu, J. (2000). Las técnicas de Análisis de Contenido: Una Revisión Actualizada. Extraído el 16 de Junio de 2011 desde http://public.centrodeestudiosandaluces.es/pdfs/S200103.pdf

Araya, S. (2002). Las Representaciones Sociales: Ejes Teóricos para su Discusión. FLACSO, Facultad Latinoamericana de Ciencias Sociales, Costa Rica, 127. Extraído el 26 de abril de 2011 desde http://www.flacso.or.cr/index.php?id=129

Bardin, L. (1986). Análisis de Contenido. Madrid. Akal.

Bernal, C. (2010) Metodología de la investigación- administración, economía, humanidades y ciencias sociales. Editorial Pesaron. Bogotá, Colombia.

Briones, G. (1998) La investigación social y educativa. Tercer mundo Editores. Bogotá, Colombia.

Colombia, Ministerio de Educación Nacional - Ministerio del Medio Ambiente. (2002). Política Nacional de Educación Ambiental (SINA). Extraído el 10 de marzo de 2011 desde http://www.areaeducada.org/area/images/documentos/politica.pdf

Cortes, C. \& Lemus, D. (2005). Representaciones sociales del concepto ambiente en los estudiantes del programa Licenciatura en Educación Básica con énfasis en Ciencias Sociales de la Universidad Pedagógica Nacional. Tesis de pregrado no publicada. Universidad Pedagógica Nacional.

Darwin, Ch: La expresión de las Emociones en los Animales y en el Hombre. Título original: The Expression of Emotions in Animals and Man. Traductor: Tomás Ramón Fernández Rodríguez. Alianza Editorial, S. A. Madrid, 1984

Flores, R. (2008). Representaciones sociales del medio ambiente. Perfiles Educativos (30)120, 33-62. Extraído el 08 de junio de 2011 en http://redalyc.uaemex.mx/pdf/132/13211159003.pdf 
Bio-grafia Escritos sobre la Biología y su Enseñanza.

Edición Extra-Ordinaria. ISSN 2027-1034 P. p 95 - 103

Memorias del VII Encuentro Nacional de Experiencias en la Enseñanza de la Biologia y la Educación Ambiental y II Congreso Nacional de Investigación en la Enseñanza de la Biología

Flores, R. (2010). Medio Ambiente y Educación Ambiental: Representaciones Sociales de los Profesores en Formación. Magis, Revista Internacional de Investigación en Educación (2)4, 401-414. Extraído el 22 de mayo de 2011 en http://www.javeriana.edu.co/magis/numero-cuatro/magis-4-art-9.html

Gadamer, H. G. (1993). Verdad y

método I. Fundamentos de una hermenéutica. Filosófica. Sígueme. Salamanca. Ediciones ALJIBE.

González, E. \& Sauvé, L. (2008). Educación, Medio Ambiente y Sustentabilidad: Once lecturas Críticas. México. Siglo XXI Editores S.A.

Heller, E. (2008) Psicología del color. Como actúan los colores sobre los sentimientos y la razón. Editorial GILI. España.

Jodelet, D. (2003) Representations

Socials: un domaine en

expansión. Les Representations

Socials, París. Presses

Universitaires de France.

Kavadlof, S. (2004). Una Biografía de

la Lluvia. Buenos Aires.

Argentina. Ensayo

Literario. Editorial Same.

Krippendorff, K. (1990). Metodología de análisis de contenido. Teoría y Práctica. España. Ediciones Paidos Ibérica.

Llanos, N \& Ruiz, L (2010) Representaciones sociales de ambiente y sus implicaciones en el desarrollo del PRAE de la comunidad educativa del colegio centro piloto de educación nueva Tibabuyes. Colombia: Universidad Distrital Francisco José de caldas facultad de Biología.

Moscovici, S. (1979). El psicoanálisis, su imagen y su público, Buenos Aires. Huemul.

Reigota, M. (1999) Las representations sociales de l'environment et les pratiques pédagogiques quotidiennes des professeurs de sciences a Sao Paulo- Brasil.

Sampieri, R \&Collado, F (1998). Metodología de la investigación. Editorial Mc Graw - Hill. México. 
Bio-grafia Escritos sobre La Biología y su Enseñanza.

Edición Extra-Ordinaria. ISSN 2027-1034 P. p 95 - 103

Memorias del VII Encuentro Nacional de Experiencias en la Enseñanza de la Biología y la Educación Ambiental y 11 Congreso Nacional de Investigación en la Enseñanza de la Biología

Sauvé, L. (1999). La Educación Ambiental entre la Modernidad y la Postmodernidad: En busca de un marco de referencia educativo integrador.

Tópicos en Educación Ambiental, 1(2), 7-26. Extraído el 16 de junio de 2011 en http://www.ambiente.gov.ar/infotecaea/descargas/sauve02.pdf 